ANNALES

POLONICI MATHEMATICI

$92.3(2007)$

\title{
Differentiable solutions for a class of functional equations
}

\author{
by V. Murugan and P. V. Subrahmanyam (Chennai)
}

Abstract. We give a set of sufficient conditions for the existence of differentiable solutions for a functional equation involving a series of iterates, using a method different from that of Baker and Zhang [Ann. Polon. Math. 73 (2000)].

1. Introduction. Iterative functional equations are functional equations involving the iterates of unknown functions. Many authors, including Abel, Babbage, Kuczma and Schröder, have contributed to this topic with a long history (see [2]). The iterative root problem is the problem of finding a function $f$ which equals a given function $F$ after $n$ iterations and it arises in the theory of dynamical systems. In this context, Zhang [9] solved the problem of finding a function $f$ such that certain convex combinations of the iterates of $f$ equal $F$, i.e.,

$$
\sum_{i=1}^{n} \lambda_{i} f^{i}(x)=F(x)
$$

This equation has been studied in a variety of ways and theorems on the existence and uniqueness of solutions have been established by many authors (see [3-9]). Baker and Zhang [1] studied (1.1) assuming $\lambda_{i}$ 's are functions of $x$ and obtained continuous solutions. In this paper, we provide sufficient conditions for the existence of differentiable solutions in the interval $I=[a, b] \subset \mathbb{R}$ for the functional equation of the form

$$
\sum_{i=1}^{\infty} \lambda_{i}(x) H_{i}\left(f^{i}(x)\right)=F(x), \quad x \in I,
$$

where $\lambda_{i}(x)$ is a sequence of nonnegative functions on $I$ such that $\sum_{i=1}^{\infty} \lambda_{i}(x)$ $=1, H_{i}$ 's and $F$ being given functions.

2000 Mathematics Subject Classification: 39B12, 47H10.

Key words and phrases: iterative functional equations, differentiable solutions, Banach's contraction principle, Schauder's fixed point theorem. 
2. Preliminaries. Consider the Banach space $C^{1}(I, \mathbb{R})$ of all continuously differentiable functions from $I$ into $\mathbb{R}$ with the norm $\|\cdot\|_{1}$, where $\|\phi\|_{1}=\|\phi\|+\left\|\phi^{\prime}\right\|$ and $\|\phi\|=\sup _{x \in I}|\phi(x)|$. For $M, M^{\prime} \geq 0$, we define

$$
\mathcal{Q}^{1}\left(M, M^{\prime}\right)=\left\{f \in C^{1}(I, \mathbb{R}):\left|f^{\prime}(x)\right| \leq M,\left|f^{\prime}(x)-f^{\prime}(y)\right| \leq M^{\prime}|x-y|\right\}
$$

and for $\delta \geq 0$,

$$
\mathcal{F}_{\delta}^{1}\left(M, M^{\prime}\right)=\left\{f \in \mathcal{Q}^{1}\left(M, M^{\prime}\right): f(a)=a, f(b)=b, \delta \leq f^{\prime}(x)\right\} .
$$

The proofs of the following propositions are easy and hence omitted.

Proposition 2.1 (see [4]). If $M<1$ or $\delta>1$ then $\mathcal{F}_{\delta}^{1}\left(M, M^{\prime}\right)$ is empty, and if $M=1$ or $\delta=1$ then $\mathcal{F}_{\delta}^{1}\left(M, M^{\prime}\right)$ is either empty or contains only the identity map.

Proposition 2.2. The set $\mathcal{F}_{\delta}^{1}\left(M, M^{\prime}\right)$ is a convex compact subset of $C^{1}(I, \mathbb{R})$.

In view of Proposition 2.1, one cannot seek solutions of equations such as (1.2) in $\mathcal{F}_{\delta}^{1}\left(M, M^{\prime}\right)$ without imposing conditions on $M$. The following lemma is essentially due to Zhang [9] and with the assumption that $M>1$.

Lemma 2.3. Let $\phi, \psi \in \mathcal{Q}^{1}\left(M, M^{\prime}\right)$. Then for $i=1,2, \ldots$,

(1) $\left|\left(\phi^{i}\right)^{\prime}(x)\right| \leq M^{i}, \quad \forall x \in I$,

(2) $\left|\left(\phi^{i}\right)^{\prime}\left(x_{1}\right)-\left(\phi^{i}\right)^{\prime}\left(x_{2}\right)\right| \leq M^{\prime} \frac{M^{i-1}}{M-1}\left(M^{i}-1\right)\left|x_{1}-x_{2}\right|, \quad \forall x_{1}, x_{2} \in I$,

(3) $\left\|\phi^{i}-\psi^{i}\right\| \leq \frac{M^{i}-1}{M-1}\|\phi-\psi\|$,

(4) $\left\|\left(\phi^{i}\right)^{\prime}-\left(\psi^{i}\right)^{\prime}\right\| \leq i M^{i-1}\left\|\phi^{\prime}-\psi^{\prime}\right\|+M^{\prime} M^{i-2}\left[M^{i}-i M+(i-1)\right]\|\phi-\psi\|$.

Lemma 2.4. For $\delta>0, M>0$ and $M^{\prime} \geq 0$, if $f \in \mathcal{F}_{\delta}^{1}\left(M, M^{\prime}\right)$, then $f$ is a diffeomorphism on $I$ onto $I$ and $f^{-1} \in \overline{\mathcal{F}}_{1 / M}^{1}\left(1 / \delta, M^{\prime} / \delta^{3}\right)$.

Proof. As $0<\delta \leq f^{\prime}(x) \leq M$, and $f(a)=a, f(b)=b, f$ is a strictly increasing function from $I$ onto itself and hence $f$ is invertible on $I$ and $f^{-1}(a)=a$ and $f^{-1}(b)=b$. As $\left(f^{-1}\right)^{\prime}(x)=1 / f^{\prime}\left(f^{-1}(x)\right)$, for $x \in I$ we have

$$
1 / M \leq\left(f^{-1}\right)^{\prime}(x) \leq 1 / \delta .
$$

As $f^{\prime}(x) \geq \delta>0$ and $\left|f^{\prime}(x)-f^{\prime}(y)\right| \leq M^{\prime}|x-y|$, for $x, y \in I$, we have

$$
\begin{aligned}
\left|\left(f^{-1}\right)^{\prime}(x)-\left(f^{-1}\right)^{\prime}(y)\right| & =\left|\frac{1}{f^{\prime}\left(f^{-1}(x)\right)}-\frac{1}{f^{\prime}\left(f^{-1}(y)\right)}\right| \\
& \leq \frac{1}{\delta^{2}}\left|f^{\prime}\left(f^{-1}(x)\right)-f^{\prime}\left(f^{-1}(y)\right)\right| .
\end{aligned}
$$

As $\left|f^{\prime}(x)-f^{\prime}(y)\right| \leq M^{\prime}|x-y|$, for $x, y \in I$ and by (2.3), we get

$$
\left|\left(f^{-1}\right)^{\prime}(x)-\left(f^{-1}\right)^{\prime}(y)\right| \leq \frac{M^{\prime}}{\delta^{2}}\left|f^{-1}(x)-f^{-1}(y)\right| \leq \frac{M^{\prime}}{\delta^{3}}|x-y| .
$$

The result follows from (2.3) and (2.4). 
LEMMA 2.5 (Zhang [9]). Let $\phi_{1}$ and $\phi_{2}$ be two homeomorphisms from I onto itself with $\left|\phi_{i}\left(x_{1}\right)-\phi_{i}\left(x_{2}\right)\right| \leq M\left|x_{1}-x_{2}\right|$ for all $x_{1}, x_{2} \in I, i=1,2$, for some $M>0$. Then

$$
\left\|\phi_{1}-\phi_{2}\right\| \leq M\left\|\phi_{1}^{-1}-\phi_{2}^{-1}\right\| .
$$

Lemma 2.6. If $f, g \in \mathcal{F}_{\delta}^{1}\left(M, M^{\prime}\right)$ where $0<\delta \leq 1 \leq M$ and $M^{\prime} \geq 0$, then

$$
\left\|\left(f^{-1}\right)^{\prime}-\left(g^{-1}\right)^{\prime}\right\| \leq \frac{M^{\prime}}{\delta^{3}}\|f-g\|+\frac{1}{\delta^{2}}\left\|f^{\prime}-g^{\prime}\right\| .
$$

Proof. As $f^{\prime}(x) \geq 0$, for $x \in I$, we get

$$
\begin{aligned}
\left|\left(f^{-1}\right)^{\prime}(x)-\left(g^{-1}\right)^{\prime}(x)\right|= & \left|\frac{1}{f^{\prime}\left(f^{-1}(x)\right)}-\frac{1}{g^{\prime}\left(g^{-1}(x)\right)}\right| \\
\leq & \frac{1}{\delta^{2}}\left|f^{\prime}\left(f^{-1}(x)\right)-g^{\prime}\left(g^{-1}(x)\right)\right| \\
\leq & \frac{1}{\delta^{2}}\left\{\left|f^{\prime}\left(f^{-1}(x)\right)-f^{\prime}\left(g^{-1}(x)\right)\right|\right. \\
& \left.+\left|f^{\prime}\left(g^{-1}(x)\right)-g^{\prime}\left(g^{-1}(x)\right)\right|\right\} \\
\leq & \frac{1}{\delta^{2}}\left\{M^{\prime}\left\|f^{-1}-g^{-1}\right\|+\left\|f^{\prime}-g^{\prime}\right\|\right\} .
\end{aligned}
$$

Using Lemma 2.5 for $f^{-1}$ and $g^{-1}$, we have

$$
\left\|\left(f^{-1}\right)^{\prime}-\left(g^{-1}\right)^{\prime}\right\| \leq \frac{M^{\prime}}{\delta^{3}}\|f-g\|+\frac{1}{\delta^{2}}\left\|f^{\prime}-g^{\prime}\right\|
$$

and thus the result follows.

Proposition 2.7. The function $f$ is a solution for the functional equation

$$
\sum_{i=1}^{\infty} \lambda_{i}(x) H_{i}\left(f^{i}(x)\right)=F(x), \quad \forall x \in[a, b],
$$

if and only if $g(x)=h^{-1}(f(h(x)))$ is a solution for the functional equation

$$
\sum_{i=1}^{\infty} \mu_{i}(x) R_{i}\left(g^{i}(x)\right)=G(x), \quad \forall x \in[0,1],
$$

where $\mu_{i}(x)=\lambda_{i}(h(x)), R_{i}(x)=h^{-1}\left(H_{i}(h(x))\right), G(x)=h^{-1}(F(h(x)))$ and $h(x)=a+x(b-a)$ for $x \in[0,1]$ with $\sum_{i=1}^{\infty} \lambda_{i}(x)=1$.

Proof. Let $f$ be a solution for (2.7). Note that $h$ and $h^{-1}$ are affine and continuous maps and $\sum_{i=1}^{\infty} \mu_{i}(x)=1$ for $x \in[0,1]$. Therefore for $x \in[0,1]$, 


$$
\begin{aligned}
\sum_{i=1}^{\infty} \mu_{i}(x) R_{i}\left(g^{i}(x)\right) & =\lim _{n \rightarrow \infty} \frac{1}{\sum_{j=1}^{n} \mu_{j}(x)} \sum_{i=1}^{n} \mu_{i}(x) R_{i}\left(g^{i}(x)\right) \\
& =\lim _{n \rightarrow \infty} \frac{1}{\sum_{j=1}^{n} \mu_{j}(x)} \sum_{i=1}^{n} \mu_{i}(x) h^{-1}\left(H_{i}\left(h\left(h^{-1} f^{i}(h(x))\right)\right)\right) \\
& =\lim _{n \rightarrow \infty} \sum_{i=1}^{n} \frac{\mu_{i}(x)}{\sum_{j=1}^{n} \mu_{j}(x)} h^{-1}\left(H_{i}\left(f^{i}(h(x))\right)\right) \\
& =\lim _{n \rightarrow \infty} h^{-1}\left[\frac{1}{\sum_{j=1}^{n} \mu_{j}(x)} \sum_{i=1}^{n} \mu_{i}(x) H_{i}\left(f^{i}(h(x))\right)\right] \\
& =h^{-1}\left[\lim _{n \rightarrow \infty} \sum_{i=1}^{n} \mu_{i}(x) H_{i}\left(f^{i}(h(x))\right)\right] \\
& =h^{-1}\left[\sum_{i=1}^{\infty} \mu_{i}(x) H_{i}\left(f^{i}(h(x))\right)\right]=h^{-1}(F(h(x))) .
\end{aligned}
$$

Thus

$$
\sum_{i=1}^{\infty} \mu_{i}(x) R_{i}\left(g^{i}(x)\right)=G(x) .
$$

The converse follows similarly.

In view of Proposition 2.7, it suffices to prove the existence of solution for the functional equation $(1.2)$ on $I=[0,1]$.

3. Existence, uniqueness and stability. We begin with a lemma concerning infinite series. Its proof is straightforward and hence omitted.

Lemma 3.1. Let $L_{i} \geq 1, \alpha_{i}, \beta_{i}, \Lambda_{i}$ and $L_{i}^{\prime}$ be nonnegative numbers for $i \in \mathbb{N}$ and $M>1$ satisfying

$$
\sum_{i=1}^{\infty} \beta_{i}<\infty, \quad \sum_{i=1}^{\infty} \alpha_{i} L_{i} M^{2 i}<\infty, \quad \sum_{i=1}^{\infty} \Lambda_{i}\left[L_{i}+L_{i}^{\prime}\right] M^{2 i}<\infty .
$$

Then for any $\delta>0$ and $M^{\prime} \geq 0$, the following series denoted by $K_{1}$ to $K_{6}$ are convergent:

$$
\begin{aligned}
& K_{1}=\sum_{i=1}^{\infty}\left\{\frac{1}{\delta} \alpha_{i}+\Lambda_{i} L_{i} M^{i-1}\right\} \\
& K_{2}=\sum_{i=1}^{\infty}\left\{\frac{1}{\delta^{2}} \beta_{i}+\frac{2}{\delta} \alpha_{i} L_{i} M^{i-1}+\Lambda_{i} L_{i}^{\prime} M^{2(i-1)}\right\},
\end{aligned}
$$




$$
\begin{aligned}
K_{3}= & \frac{1}{\delta^{3}} \sum_{i=1}^{\infty} \alpha_{i}+\sum_{i=1}^{\infty} \Lambda_{i} L_{i} M^{i-2} \frac{M^{i-1}-1}{M-1}, \\
K_{4}= & \frac{1}{\delta} \sum_{i=1}^{\infty} \alpha_{i}+\sum_{i=1}^{\infty} \Lambda_{i} L_{i} \frac{M^{i-1}-1}{M-1}, \\
K_{5}= & \frac{1}{\delta^{2}} \sum_{i=1}^{\infty} \beta_{i}+\frac{1}{\delta} \sum_{i=1}^{\infty} \alpha_{i} L_{i} \frac{M^{i-1}-1}{M-1}+\frac{1}{\delta} \sum_{i=1}^{\infty} \alpha_{i} L_{i} M^{i-1} \\
& +\sum_{i=1}^{\infty} \Lambda_{i} L_{i}^{\prime} M^{i-1} \frac{M^{i-1}-1}{M-1} \\
& +M^{\prime}\left\{\frac{1}{\delta^{3}} \sum_{i=1}^{\infty} \alpha_{i}+\sum_{i=1}^{\infty} \Lambda_{i} L_{i} M^{i-3}\left[M^{i-1}-(i-1) M+(i-2)\right]\right\}, \\
K_{6}= & \frac{1}{\delta^{2}} \sum_{i=1}^{\infty} \alpha_{i}+\sum_{i=1}^{\infty} \Lambda_{i} L_{i}(i-1) M^{i-2} .
\end{aligned}
$$

Now we state our main result on the existence of a solution to (1.2).

THEOREM 3.2. In addition to the hypotheses of Lemma 3.1, let $\lambda_{i}$ be a sequence of nonnegative functions on $I$ such that $\lambda_{i}(x) \in \mathcal{Q}^{1}\left(\alpha_{i}, \beta_{i}\right)$ and $\gamma_{i} \leq \lambda_{i}(x) \leq \Lambda_{i}$ for $i=1,2, \ldots$, and $\sum_{i=1}^{\infty} \lambda_{i}(x)=1$ for $x \in$ I. Let $H_{i} \in \mathcal{F}_{l_{i}}^{1}\left(L_{i}, L_{i}^{\prime}\right)$ where $l_{i}$ are nonnegative numbers for $i=1,2, \ldots$ Suppose $0<\delta<1, M^{*} \geq 0$, and

$$
K_{0}=\sum_{i=1}^{\infty} \gamma_{i} l_{i} \delta^{i-1}-\frac{1}{\delta} \sum_{i=1}^{\infty} \alpha_{i}>M^{2} K_{3} .
$$

Then for any function $F$ in $\mathcal{F}_{K_{1} \delta}^{1}\left(K_{0} M, M^{*}\right)$, the functional equation

$$
\sum_{i=1}^{\infty} \lambda_{i}(x) H_{i}\left(f^{i}(x)\right)=F(x)
$$

has a solution $f$ in $\mathcal{F}_{\delta}^{1}\left(M, M^{\prime}\right)$ for every $M^{\prime} \geq\left(M^{*}+M^{2} K_{2}\right) /\left(K_{0}-M^{2} K_{3}\right)$.

The following lemmata will lead directly to the proof of Theorem 3.2. For $f \in \mathcal{F}_{\delta}^{1}\left(M, M^{\prime}\right)$, we define $L_{f}: I \rightarrow I$ by

$$
L_{f}(x)=\sum_{i=1}^{\infty} \lambda_{i}\left(f^{-1}(x)\right) H_{i}\left(f^{i-1}(x)\right), \quad x \in I .
$$

LEMMA 3.3. In addition to the hypotheses of Theorem 3.2, suppose that $f \in \mathcal{F}_{\delta}^{1}\left(M, M^{\prime}\right)$. Then $L_{f} \in \mathcal{F}_{K_{0}}^{1}\left(K_{1}, K_{2}+M^{\prime} K_{3}\right)$. 
Proof. It is clear that $L_{f}(0)=0$ and $L_{f}(1)=1$. Because $K_{1}=\sum_{i=1}^{\infty}\left\{\delta^{-1} \alpha_{i}\right.$ $\left.+\Lambda_{i} L_{i} M^{i-1}\right\}<\infty$, the function $L_{f}$ is differentiable and

$$
\begin{aligned}
L_{f}^{\prime}(x)= & \sum_{i=1}^{\infty} \lambda_{i}^{\prime}\left(f^{-1}(x)\right)\left(f^{-1}\right)^{\prime}(x) H_{i}\left(f^{i-1}(x)\right) \\
& +\sum_{i=1}^{\infty} \lambda_{i}\left(f^{-1}(x)\right) H_{i}^{\prime}\left(f^{i-1}(x)\right)\left(f^{i-1}\right)^{\prime}(x)
\end{aligned}
$$

and by the hypothesis of Theorem 3.2, we have

$$
0<K_{0} \leq L_{f}^{\prime}(x) \leq K_{1} .
$$

Thus

$$
1 / K_{1} \leq\left(L_{f}^{-1}\right)^{\prime}(x) \leq 1 / K_{0} .
$$

From the definition of $L_{f}(x)$ and the triangle inequality, for $x, y \in I$,

$$
\begin{aligned}
& \left|L_{f}^{\prime}(x)-L_{f}^{\prime}(y)\right| \\
& \leq \sum_{i=1}^{\infty}\left\{\mid \lambda_{i}^{\prime}\left(f^{-1}(x)\right)\left(f^{-1}\right)^{\prime}(x) H_{i}\left(f^{i-1}(x)\right)+\lambda_{i}\left(f^{-1}(x)\right) H_{i}^{\prime}\left(f^{i-1}(x)\right)\left(f^{i-1}\right)^{\prime}(x)\right. \\
& \left.-\lambda_{i}^{\prime}\left(f^{-1}(y)\right)\left(f^{-1}\right)^{\prime}(y) H_{i}\left(f^{i-1}(y)\right)-\lambda_{i}\left(f^{-1}(y)\right) H_{i}^{\prime}\left(f^{i-1}(y)\right)\left(f^{i-1}\right)^{\prime}(y) \mid\right\} \\
& \leq \sum_{i=1}^{\infty}\left\{\left|\lambda_{i}^{\prime}\left(f^{-1}(x)\right)\left(f^{-1}\right)^{\prime}(x) H_{i}\left(f^{i-1}(x)\right)-\lambda_{i}^{\prime}\left(f^{-1}(y)\right)\left(f^{-1}\right)^{\prime}(y) H_{i}\left(f^{i-1}(y)\right)\right|\right. \\
& \left.+\left|\lambda_{i}\left(f^{-1}(x)\right) H_{i}^{\prime}\left(f^{i-1}(x)\right)\left(f^{i-1}\right)^{\prime}(x)-\lambda_{i}\left(f^{-1}(y)\right) H_{i}^{\prime}\left(f^{i-1}(y)\right)\left(f^{i-1}\right)^{\prime}(y)\right|\right\} \\
& =: \sum_{i=1}^{\infty}\left(A_{i}+B_{i}\right) \text {. }
\end{aligned}
$$

By Lemma 2.4, $H_{i}(x) \leq 1$ and by the definition of $\lambda_{i}(x)$ and $H_{i}(x)$, for all $i$,

$$
\begin{aligned}
A_{i} \leq & \left|\lambda_{i}^{\prime}\left(f^{-1}(x)\right)\left(f^{-1}\right)^{\prime}(x) H_{i}\left(f^{i-1}(x)\right)-\lambda_{i}^{\prime}\left(f^{-1}(y)\right)\left(f^{-1}\right)^{\prime}(y) H_{i}\left(f^{i-1}(x)\right)\right| \\
& +\left|\lambda_{i}^{\prime}\left(f^{-1}(y)\right)\left(f^{-1}\right)^{\prime}(y) H_{i}\left(f^{i-1}(x)\right)-\lambda_{i}^{\prime}\left(f^{-1}(y)\right)\left(f^{-1}\right)^{\prime}(y) H_{i}\left(f^{i-1}(y)\right)\right| \\
\leq & \left|\lambda_{i}^{\prime}\left(f^{-1}(x)\right)\left(f^{-1}\right)^{\prime}(x)-\lambda_{i}^{\prime}\left(f^{-1}(y)\right)\left(f^{-1}\right)^{\prime}(y)\right| \\
& +\frac{1}{\delta} \alpha_{i}\left|H_{i}\left(f^{i-1}(x)\right)-H_{i}\left(f^{i-1}(y)\right)\right| \\
\leq & \left|\lambda_{i}^{\prime}\left(f^{-1}(x)\right)-\lambda_{i}^{\prime}\left(f^{-1}(y)\right)\right|\left|\left(f^{-1}\right)^{\prime}(x)\right| \\
& +\left|\lambda_{i}^{\prime}\left(f^{-1}(y)\right)\right|\left|\left(f^{-1}\right)^{\prime}(x)-\left(f^{-1}\right)^{\prime}(y)\right|+\frac{1}{\delta} \alpha_{i} L_{i}\left|f^{i-1}(x)-f^{i-1}(y)\right| .
\end{aligned}
$$


In view of Lemmas 2.3 and 2.4 , we get

$$
\begin{aligned}
A_{i} & \leq \frac{1}{\delta} \beta_{i}\left|f^{-1}(x)-f^{-1}(y)\right|+\frac{M^{\prime}}{\delta^{3}} \alpha_{i}|x-y|+\frac{1}{\delta} \alpha_{i} L_{i} M^{i-1}|x-y| \\
& \leq\left\{\frac{1}{\delta^{2}} \beta_{i}+\frac{M^{\prime}}{\delta^{3}} \alpha_{i}+\frac{1}{\delta} \alpha_{i} L_{i} M^{i-1}\right\}|x-y| .
\end{aligned}
$$

Again for each $i$,

$$
\begin{aligned}
B_{i} \leq & \left|\lambda_{i}\left(f^{-1}(x)\right) H_{i}^{\prime}\left(f^{i-1}(x)\right)-\lambda_{i}\left(f^{-1}(y)\right) H_{i}^{\prime}\left(f^{i-1}(y)\right)\right|\left|\left(f^{i-1}\right)^{\prime}(x)\right| \\
& +\left|\lambda_{i}\left(f^{-1}(y)\right)\right|\left|H_{i}^{\prime}\left(f^{i-1}(y)\right)\right|\left|\left(f^{i-1}\right)^{\prime}(x)-\left(f^{i-1}\right)^{\prime}(y)\right| .
\end{aligned}
$$

By (1) and (2) of Lemma 2.3 and definitions of $\lambda_{i}(x)$ and $H_{i}(x)$, we get

$$
\begin{aligned}
B_{i} \leq & M^{i-1}\left|\lambda_{i}\left(f^{-1}(x)\right) H_{i}^{\prime}\left(f^{i-1}(x)\right)-\lambda_{i}\left(f^{-1}(y)\right) H_{i}^{\prime}\left(f^{i-1}(y)\right)\right| \\
& +\Lambda_{i} L_{i}\left|\left(f^{i-1}\right)^{\prime}(x)-\left(f^{i-1}\right)^{\prime}(y)\right| \\
\leq & M^{i-1}\left\{\left|\lambda_{i}\left(f^{-1}(x)\right)-\lambda_{i}\left(f^{-1}(y)\right)\right|\left|H_{i}^{\prime}\left(f^{i-1}(x)\right)\right|\right. \\
& \left.+\left|\lambda_{i}\left(f^{-1}(y)\right)\right|\left|H_{i}^{\prime}\left(f^{i-1}(x)\right)-H_{i}^{\prime}\left(f^{i-1}(y)\right)\right|\right\} \\
& +M^{\prime} \Lambda_{i} L_{i} M^{i-2} \frac{M^{i-1}-1}{M-1}|x-y| .
\end{aligned}
$$

Using the definitions of $\lambda_{i}(x)$ and $H_{i}(x)$, and Lemmas 2.3 and 2.4, we get

$$
\begin{aligned}
B_{i} \leq & \alpha_{i} L_{i} M^{i-1}\left|f^{-1}(x)-f^{-1}(y)\right|+\Lambda_{i} L_{i}^{\prime} M^{i-1}\left|f^{i-1}(x)-f^{i-1}(y)\right| \\
& +M^{\prime} \Lambda_{i} L_{i} M^{i-2} \frac{M^{i-1}-1}{M-1}|x-y| \\
\leq & \frac{1}{\delta} \alpha_{i} L_{i} M^{i-1}|x-y|+\Lambda_{i} L_{i}^{\prime} M^{2(i-1)}|x-y| \\
& +M^{\prime} \Lambda_{i} L_{i} M^{i-2} \frac{M^{i-1}-1}{M-1}|x-y| .
\end{aligned}
$$

From (3.13) and (3.14), for $x, y \in I$ we get

$$
\left|L_{f}^{\prime}(x)-L_{f}^{\prime}(y)\right| \leq\left(K_{2}+M^{\prime} K_{3}\right)|x-y| .
$$

Thus, by (3.11) and (3.15), $L_{f}$ is in $\mathcal{F}_{K_{0}}^{1}\left(K_{1}, K_{2}+M^{\prime} K_{3}\right)$.

LEMMA 3.4. In addition to the hypotheses of Theorem 3.2, suppose that $f, g \in \mathcal{F}_{\delta}^{1}\left(M, M^{\prime}\right)$. Then

(i) $\left\|L_{f}-L_{g}\right\| \leq K_{4}\|f-g\|$,

(ii) $\left\|L_{f}^{-1}-L_{g}^{-1}\right\| \leq \frac{K_{4}}{K_{0}}\|f-g\|$,

(iii) $\left\|L_{f}^{\prime}-L_{g}^{\prime}\right\| \leq K_{5}\|f-g\|+K_{6}\left\|f^{\prime}-g^{\prime}\right\|$,

(iv) $\left\|\left(L_{f}^{-1}\right)^{\prime}-\left(L_{g}^{-1}\right)^{\prime}\right\| \leq\left[\frac{K_{4}}{K_{0}^{3}}\left(K_{2}+M^{\prime} K_{3}\right)+\frac{K_{5}}{K_{0}^{2}}\right]\|f-g\|+\frac{K_{6}}{K_{0}^{2}}\left\|f^{\prime}-g^{\prime}\right\|$. 
Proof. For $x \in I$ and $f, g \in \mathcal{F}_{\delta}^{1}\left(M, M^{\prime}\right)$,

$$
\begin{aligned}
\left|L_{f}(x)-L_{g}(x)\right| \leq & \sum_{i=1}^{\infty}\left|\lambda_{i}\left(f^{-1}(x)\right) H_{i}\left(f^{i-1}(x)\right)-\lambda_{i}\left(g^{-1}(x)\right) H_{i}\left(g^{i-1}(x)\right)\right| \\
\leq & \sum_{i=1}^{\infty}\left\{\left|\lambda_{i}\left(f^{-1}(x)\right)-\lambda_{i}\left(g^{-1}(x)\right)\right|\left|H_{i}\left(f^{i-1}(x)\right)\right|\right. \\
& \left.+\left|\lambda_{i}\left(g^{-1}(x)\right)\right|\left|H_{i}\left(f^{i-1}(x)\right)-H_{i}\left(g^{i-1}(x)\right)\right|\right\} .
\end{aligned}
$$

From Lemmas 2.3 and 2.5 and the definitions of $\lambda_{i}(x)$ and $H_{i}(x)$, we get

$$
\begin{aligned}
\left|L_{f}(x)-L_{g}(x)\right| & \leq \sum_{i=1}^{\infty}\left\{\alpha_{i}\left|f^{-1}(x)-g^{-1}(x)\right|+\Lambda_{i} L_{i}\left\|f^{i-1}-g^{i-1}\right\|\right\} \\
& \leq \sum_{i=1}^{\infty}\left\{\frac{1}{\delta} \alpha_{i}+\Lambda_{i} L_{i} \frac{M^{i-1}-1}{M-1}\right\}\|f-g\| .
\end{aligned}
$$

Therefore,

$$
\left\|L_{f}-L_{g}\right\| \leq K_{4}\|f-g\|,
$$

proving (i). By (3.12), for $f \in \mathcal{F}_{\delta}^{1}\left(M, M^{\prime}\right)$,

$$
\left|L_{f}^{-1}(x)-L_{f}^{-1}(y)\right| \leq \frac{1}{K_{0}}|x-y| .
$$

So, from Lemma 2.5, and (3.16) we get

$$
\left\|L_{f}^{-1}-L_{g}^{-1}\right\| \leq \frac{1}{K_{0}}\left\|L_{f}-L_{g}\right\| \leq \frac{K_{4}}{K_{0}}\|f-g\|,
$$

proving (ii). Now for $x \in I$,

$$
\begin{aligned}
& \left|L_{f}^{\prime}(x)-L_{g}^{\prime}(x)\right| \\
& \leq \sum_{i=1}^{\infty}\left\{\mid \lambda_{i}^{\prime}\left(f^{-1}(x)\right)\left(f^{-1}\right)^{\prime}(x) H_{i}\left(f^{i-1}(x)\right)+\lambda_{i}\left(f^{-1}(x)\right) H_{i}^{\prime}\left(f^{i-1}(x)\right)\left(f^{i-1}\right)^{\prime}(x)\right. \\
& \left.\quad-\lambda_{i}^{\prime}\left(g^{-1}(x)\right)\left(g^{-1}\right)^{\prime}(x) H_{i}\left(g^{i-1}(x)\right)-\lambda_{i}\left(g^{-1}(x)\right) H_{i}^{\prime}\left(g^{i-1}(x)\right)\left(g^{i-1}\right)^{\prime}(x) \mid\right\} \\
& \leq \sum_{i=1}^{\infty}\left\{\left|\lambda_{i}^{\prime}\left(f^{-1}(x)\right)\left(f^{-1}\right)^{\prime}(x) H_{i}\left(f^{i-1}(x)\right)-\lambda_{i}^{\prime}\left(g^{-1}(x)\right)\left(g^{-1}\right)^{\prime}(x) H_{i}\left(g^{i-1}(x)\right)\right|\right. \\
& \left.\quad+\left|\lambda_{i}\left(f^{-1}(x)\right) H_{i}^{\prime}\left(f^{i-1}(x)\right)\left(f^{i-1}\right)^{\prime}(x)-\lambda_{i}\left(g^{-1}(x)\right) H_{i}^{\prime}\left(g^{i-1}(x)\right)\left(g^{i-1}\right)^{\prime}(x)\right|\right\} \\
& =: \sum_{i=1}^{\infty}\left(C_{i}+D_{i}\right) .
\end{aligned}
$$


Using Lemmas 2.3 and 2.4, for each $i$ we get

$$
\begin{aligned}
C_{i} \leq & \left|\lambda_{i}^{\prime}\left(f^{-1}(x)\right)\left(f^{-1}\right)^{\prime}(x)-\lambda_{i}^{\prime}\left(g^{-1}(x)\right)\left(g^{-1}\right)^{\prime}(x)\right|\left|H_{i}\left(f^{i-1}(x)\right)\right| \\
& +\left|\lambda_{i}^{\prime}\left(g^{-1}(x)\right)\left(g^{-1}\right)^{\prime}(x)\right|\left|H_{i}\left(f^{i-1}(x)\right)-H_{i}\left(g^{i-1}(x)\right)\right| \\
\leq & \left|\lambda_{i}^{\prime}\left(f^{-1}(x)\right)-\lambda_{i}^{\prime}\left(g^{-1}(x)\right)\right|\left|\left(f^{-1}\right)^{\prime}(x)\right| \\
& +\left|\lambda_{i}^{\prime}\left(g^{-1}(x)\right)\right|\left|\left(f^{-1}\right)^{\prime}(x)-\left(g^{-1}\right)^{\prime}(x)\right| \\
& +\frac{1}{\delta} \alpha_{i} L_{i} \frac{M^{i-1}-1}{M-1}\|f-g\| \\
\leq & \frac{1}{\delta^{2}} \beta_{i}\|f-g\|+\frac{M^{\prime}}{\delta^{3}} \alpha_{i}\|f-g\|+\frac{1}{\delta^{2}} \alpha_{i}\left\|f^{\prime}-g^{\prime}\right\| \\
& +\frac{1}{\delta} \alpha_{i} L_{i} \frac{M^{i-1}-1}{M-1}\|f-g\| .
\end{aligned}
$$

Further, for each $i$,

$$
\begin{aligned}
D_{i}= & \left|\lambda_{i}\left(f^{-1}(x)\right) H_{i}^{\prime}\left(f^{i-1}(x)\right)\left(f^{i-1}\right)^{\prime}(x)-\lambda_{i}\left(g^{-1}(x)\right) H_{i}^{\prime}\left(g^{i-1}(x)\right)\left(g^{i-1}\right)^{\prime}(x)\right| \\
\leq & \left|\lambda_{i}\left(f^{-1}(x)\right)-\lambda_{i}\left(g^{-1}(x)\right)\right|\left|H_{i}^{\prime}\left(f^{i-1}(x)\right)\right|\left|\left(f^{i-1}\right)^{\prime}(x)\right| \\
& +\left|\lambda_{i}\left(g^{-1}(x)\right)\right|\left|H_{i}^{\prime}\left(f^{i-1}(x)\right)\left(f^{i-1}\right)^{\prime}(x)-H_{i}^{\prime}\left(g^{i-1}(x)\right)\left(g^{i-1}\right)^{\prime}(x)\right| \\
\leq & M^{i-1}\left\{\left|\lambda_{i}\left(f^{-1}(x)\right)-\lambda_{i}\left(g^{-1}(x)\right)\right|\left|H_{i}^{\prime}\left(f^{i-1}(x)\right)\right|\right. \\
& \left.+\left|\lambda_{i}\left(g^{-1}(x)\right)\right|\left|H_{i}^{\prime}\left(f^{i-1}(x)\right)-H_{i}^{\prime}\left(g^{i-1}(x)\right)\right|\right\}+\Lambda_{i} L_{i}\left\|\left(f^{i-1}\right)^{\prime}-\left(g^{i-1}\right)^{\prime}\right\| .
\end{aligned}
$$

Applying Lemma 2.3, we get

$$
\begin{aligned}
D_{i} \leq & \frac{1}{\delta} \alpha_{i} L_{i} M^{i-1}\|f-g\|+\Lambda_{i} L_{i}^{\prime} M^{i-1}\left\|f^{i-1}-g^{i-1}\right\| \\
& +\Lambda_{i} L_{i}(i-1) M^{i-2}\left\|f^{\prime}-g^{\prime}\right\| \\
& +M^{\prime} \Lambda_{i} L_{i} M^{i-3}\left[M^{i-1}-(i-1) M+(i-2)\right]\|f-g\| \\
\leq & \frac{1}{\delta} \alpha_{i} L_{i} M^{i-1}\|f-g\|+\Lambda_{i} L_{i}^{\prime} M^{i-1} \frac{M^{i-1}-1}{M-1}\|f-g\| \\
& +\Lambda_{i} L_{i}(i-1) M^{i-2}\left\|f^{\prime}-g^{\prime}\right\| \\
& +M^{\prime} \Lambda_{i} L_{i} M^{i-3}\left[M^{i-1}-(i-1) M+(i-2)\right]\|f-g\| .
\end{aligned}
$$

Using (3.19) and (3.20), we get

$$
\left|L_{f}^{\prime}(x)-L_{g}^{\prime}(x)\right| \leq K_{5}\|f-g\|+K_{6}\left\|f^{\prime}-g^{\prime}\right\| .
$$

Thus (iii) is established.

For $f, g \in \mathcal{F}_{\delta}^{1}\left(M, M^{\prime}\right)$ and $x \in I$,

$$
\left|\left(L_{f}^{-1}\right)^{\prime}(x)-\left(L_{g}^{-1}\right)^{\prime}(x)\right|=\left|\frac{1}{L_{f}^{\prime}\left(L_{f}^{-1}(x)\right)}-\frac{1}{\left(L_{g}^{\prime}\left(L_{g}^{-1}(x)\right)\right.}\right| .
$$


Using (3.11) we have

$$
\begin{aligned}
\left|\left(L_{f}^{-1}\right)^{\prime}(x)-\left(L_{g}^{-1}\right)^{\prime}(x)\right| \leq & \frac{1}{K_{0}^{2}}\left|L_{f}^{\prime}\left(L_{f}^{-1}(x)\right)-L_{g}^{\prime}\left(L_{g}^{-1}(x)\right)\right| \\
\leq & \frac{1}{K_{0}^{2}}\left\{\left|L_{f}^{\prime}\left(L_{f}^{-1}(x)\right)-L_{g}^{\prime}\left(L_{f}^{-1}(x)\right)\right|\right. \\
& \left.+\left|L_{g}^{\prime}\left(L_{f}^{-1}(x)\right)-L_{g}^{\prime}\left(L_{g}^{-1}(x)\right)\right|\right\} .
\end{aligned}
$$

From (3.15) and (3.21) we have

$$
\begin{aligned}
\left|\left(L_{f}^{-1}\right)^{\prime}(x)-\left(L_{g}^{-1}\right)^{\prime}(x)\right| \leq & \frac{1}{K_{0}^{2}}\left\{K_{5}\|f-g\|+K_{6}\left\|f^{\prime}-g^{\prime}\right\|\right. \\
& \left.+\left(K_{2}+M^{\prime} K_{3}\right)\left\|L_{f}^{-1}-L_{g}^{-1}\right\|\right\} .
\end{aligned}
$$

Further using (3.18) to estimate $\left\|L_{f}^{-1}-L_{g}^{-1}\right\|$, we get

$$
\begin{aligned}
\left\|\left(L_{f}^{-1}\right)^{\prime}-\left(L_{g}^{-1}\right)^{\prime}\right\| \leq & \frac{1}{K_{0}^{2}}\left\{K_{5}\|f-g\|+K_{6}\left\|f^{\prime}-g^{\prime}\right\|\right. \\
& \left.+\frac{K_{4}}{K_{0}}\left(K_{2}+M^{\prime} K_{3}\right)\|f-g\|\right\},
\end{aligned}
$$

thus proving (iv) of Lemma 3.4 as well.

Proof of Theorem 3.2. Define $T: \mathcal{F}_{\delta}^{1}\left(M, M^{\prime}\right) \rightarrow C^{1}(I, \mathbb{R})$ by

$$
T f(x)=\left(L_{f}^{-1}\right)(F(x)) \quad \text { for } f \in \mathcal{F}_{\delta}^{1}\left(M, M^{\prime}\right) .
$$

By the definitions of $L_{f}$ and $F$ we get $T f(0)=0$ and $T f(1)=1$. Now

$$
T f^{\prime}(x)=\left(L_{f}^{-1}\right)^{\prime}(F(x)) F^{\prime}(x)
$$

and so $\delta=\frac{1}{K_{1}} K_{1} \delta \leq T f^{\prime}(x) \leq \frac{1}{K_{0}} K_{0} M=M$. Hence

$$
\delta \leq T f^{\prime}(x) \leq M
$$

For $x, y$ in $I$,

$$
\begin{aligned}
\left|T f^{\prime}(x)-T f^{\prime}(y)\right| \leq & \left|\left(L_{f}^{-1}\right)^{\prime}(F(x)) F^{\prime}(x)-\left(L_{f}^{-1}\right)^{\prime}(F(y)) F^{\prime}(y)\right| \\
\leq & \left|\left(L_{f}^{-1}\right)^{\prime}(F(x))-\left(L_{f}^{-1}\right)^{\prime}(F(y))\right|\left|F^{\prime}(x)\right| \\
& +\left|\left(L_{f}^{-1}\right)^{\prime}(F(y))\right|\left|F^{\prime}(x)-F^{\prime}(y)\right| .
\end{aligned}
$$

Applying Lemma 2.4 for $L_{f} \in \mathcal{F}_{K_{0}}^{1}\left(K_{1}, K_{2}+M^{\prime} K_{3}\right)$ and using the definition of $F$, we get

$$
\begin{aligned}
\left|T f^{\prime}(x)-T f^{\prime}(y)\right| & \leq \frac{K_{2}+M^{\prime} K_{3}}{K_{0}^{3}} K_{0} M|F(x)-F(y)|+\frac{M^{*}}{K_{0}}|x-y| \\
& \leq \frac{M^{2}\left(K_{2}+M^{\prime} K_{3}\right)+M^{*}}{K_{0}}|x-y| .
\end{aligned}
$$


As $M^{\prime} \geq\left(M^{*}+M^{2} K_{2}\right) /\left(K_{0}-M^{2} K_{3}\right)$, we have

$$
\left|T f^{\prime}(x)-T f^{\prime}(y)\right| \leq M^{\prime}|x-y| \text {. }
$$

Hence $T$ is a self-map of $\mathcal{F}_{\delta}^{1}\left(M, M^{\prime}\right)$.

We now prove that $T$ is continuous. For $f, g \in \mathcal{F}_{\delta}^{1}\left(M, M^{\prime}\right)$ and $x \in I$,

$$
\begin{aligned}
|T f(x)-T g(x)| & =\left|L_{f}^{-1}(F(x))-L_{g}^{-1}(F(x))\right| \\
& \leq\left\|L_{f}^{-1}-L_{g}^{-1}\right\| \leq \frac{1}{K_{0}}\left\|L_{f}-L_{g}\right\| .
\end{aligned}
$$

So, by Lemma 3.4, we have

$$
\|T f-T g\| \leq \frac{K_{4}}{K_{0}}\|f-g\|
$$

and

$$
\begin{aligned}
\left|T f^{\prime}(x)-T g^{\prime}(x)\right| & =\left|\left(L_{f}^{-1}\right)^{\prime}(F(x)) F^{\prime}(x)-\left(L_{g}^{-1}\right)^{\prime}(F(x)) F^{\prime}(x)\right| \\
& \leq K_{0} M\left|\left(L_{f}^{-1}\right)^{\prime}(F(x))-\left(L_{g}^{-1}\right)^{\prime}(F(x))\right| .
\end{aligned}
$$

Using Lemma 3.4(iv), we get

$$
\begin{aligned}
\mid T f^{\prime}(x) & -T g^{\prime}(x) \mid \\
& \leq K_{0} M\left\{\frac{K_{4}\left(K_{2}+M^{\prime} K_{3}\right)}{K_{0}^{3}}\|f-g\|+\frac{K_{5}}{K_{0}^{2}}\|f-g\|+\frac{K_{6}}{K_{0}^{2}}\left\|f^{\prime}-g^{\prime}\right\|\right\} .
\end{aligned}
$$

Therefore,

$$
\begin{aligned}
& \left\|T f^{\prime}-T g^{\prime}\right\| \\
& \leq \frac{M K_{4}\left(K_{2}+M^{\prime} K_{3}\right)}{K_{0}^{2}}\|f-g\|+\frac{M K_{5}}{K_{0}}\|f-g\|+\frac{M K_{6}}{K_{0}}\left\|f^{\prime}-g^{\prime}\right\| .
\end{aligned}
$$

Consequently, from (3.26) and (3.27), we have

$$
\begin{aligned}
\|T f-T g\|_{1}= & \|T f-T g\|+\left\|T f^{\prime}-T g^{\prime}\right\| \\
\leq & \frac{K_{4}}{K_{0}}\|f-g\|+\frac{M K_{4}}{K_{0}^{2}}\left(K_{2}+M^{\prime} K_{3}\right)\|f-g\| \\
& +\frac{M K_{5}}{K_{0}}\|f-g\|+\frac{M K_{6}}{K_{0}}\left\|f^{\prime}-g^{\prime}\right\| .
\end{aligned}
$$

Hence

$$
\|T f-T g\|_{1} \leq \varrho\|f-g\|_{1}
$$

where

$$
\varrho=\max \left\{\frac{K_{4}}{K_{0}}+\frac{M K_{4}}{K_{0}^{2}}\left(K_{2}+M^{\prime} K_{3}\right)+\frac{M K_{5}}{K_{0}}, \frac{M K_{6}}{K_{0}}\right\} .
$$

This proves that $T$ is continuous. By Proposition 2.2, $T$ is a continuous self-map of the convex, compact subset $\mathcal{F}_{\delta}^{1}\left(M, M^{\prime}\right)$ of $C^{1}(I, \mathbb{R})$. So, by 
Schauder's fixed point theorem, $T$ has a fixed point, which is a solution of the functional equation (1.2).

REMARK 3.5. Clearly, by the hypothesis of Theorem $3.2, \gamma_{1}$ and $l_{1}$ are positive.

TheOREM 3.6. In addition to the hypotheses of Theorem 3.2, let

$$
\varrho=\max \left\{\frac{K_{4}}{K_{0}}+\frac{M K_{4}}{K_{0}^{2}}\left(K_{2}+M^{\prime} K_{3}\right)+\frac{M K_{5}}{K_{0}}, \frac{M K_{6}}{K_{0}}\right\}<1 .
$$

Then for $F \in \mathcal{F}_{K_{1} \delta}^{1}\left(K_{0} M, M^{*}\right)$, the functional equation (1.2) has a unique solution $f$ in $\mathcal{F}_{\delta}^{1}\left(M, M^{\prime}\right)$ and the solution $f$ depends continuously on the function $F$.

Proof. As $\varrho<1$, the uniqueness of the solution follows from the Banach contraction principle for $T$. Let $F, G \in \mathcal{F}_{K_{1} \delta}^{1}\left(K_{0} M, M^{*}\right)$, and let $f$ and $g$ be the solutions for the functional equations involving $F$ and $G$ respectively. Thus $L_{f}^{-1}(F(x))=f(x)$ and $L_{g}^{-1}(G(x))=g(x)$ for $x \in I$. So,

$$
\begin{aligned}
|f(x)-g(x)| & =\left|L_{f}^{-1}(F(x))-L_{g}^{-1}(G(x))\right| \\
& \leq\left|L_{f}^{-1}(F(x))-L_{g}^{-1}(F(x))\right|+\left|L_{g}^{-1}(F(x))-L_{g}^{-1}(G(x))\right| \\
& \leq\left\|L_{f}^{-1}-L_{g}^{-1}\right\|+\frac{1}{K_{0}}\|F-G\| .
\end{aligned}
$$

Using Lemma 3.4, we get

$$
\|f-g\| \leq \frac{K_{4}}{K_{0}}\|f-g\|+\frac{1}{K_{0}}\|F-G\| .
$$

From (3.12) and the definition of $F$, for $x$ in $I$, we have

$$
\begin{aligned}
\left|f^{\prime}(x)-g^{\prime}(x)\right|= & \left|\left(L_{f}^{-1}\right)^{\prime}(F(x)) F^{\prime}(x)-\left(L_{g}^{-1}\right)^{\prime}(G(x)) G^{\prime}(x)\right| \\
\leq & \left|\left(L_{f}^{-1}\right)^{\prime}(F(x))-\left(L_{g}^{-1}\right)^{\prime}(G(x))\right|\left|F^{\prime}(x)\right| \\
& +\left|\left(L_{g}^{-1}\right)^{\prime}(G(x))\right|\left|F^{\prime}(x)-G^{\prime}(x)\right| \\
\leq & K_{0} M\left\{\left|\left(L_{f}^{-1}\right)^{\prime}(F(x))-\left(L_{g}^{-1}\right)^{\prime}(F(x))\right|\right. \\
& \left.+\left|\left(L_{g}^{-1}\right)^{\prime}(F(x))-\left(L_{g}^{-1}\right)^{\prime}(G(x))\right|\right\}+\frac{1}{K_{0}}\left|F^{\prime}(x)-G^{\prime}(x)\right| .
\end{aligned}
$$

By Lemmas 3.3 and 3.4(iv), we get

$$
\begin{aligned}
\left\|f^{\prime}-g^{\prime}\right\| \leq & K_{0} M\left\{\left\|\left(L_{f}^{-1}\right)^{\prime}-\left(L_{g}^{-1}\right)^{\prime}\right\|+\frac{K_{2}+M^{\prime} K_{3}}{K_{0}^{3}}\|F-G\|\right\} \\
& +\frac{1}{K_{0}}\left\|F^{\prime}-G^{\prime}\right\|
\end{aligned}
$$




$$
\begin{aligned}
\leq & K_{0} M\left\{\frac{K_{4}}{K_{0}^{3}}\left(K_{2}+M^{\prime} K_{3}\right)\|f-g\|+\frac{K_{5}}{K_{0}^{2}}\|f-g\|+\frac{K_{6}}{K_{0}^{2}}\left\|f^{\prime}-g^{\prime}\right\|\right\} \\
& +\frac{K_{0} M}{K_{0}^{3}}\left(K_{2}+M^{\prime} K_{3}\right)\|F-G\|+\frac{1}{K_{0}}\left\|F^{\prime}-G^{\prime}\right\| .
\end{aligned}
$$

Thus we have

$$
\begin{aligned}
\left\|f^{\prime}-g^{\prime}\right\| \leq & \frac{M}{K_{0}}\left\{K_{5}\|f-g\|+K_{6}\left\|f^{\prime}-g^{\prime}\right\|+\frac{K_{2}+M^{\prime} K_{3}}{K_{0}}\|F-G\|\right. \\
& \left.+\frac{K_{4}\left(K_{2}+M^{\prime} K_{3}\right)}{K_{0}}\|f-g\|\right\}+\frac{1}{K_{0}}\left\|F^{\prime}-G^{\prime}\right\| .
\end{aligned}
$$

From (3.29) and (3.30) we get

$$
\begin{aligned}
\|f-g\|_{1} \leq & \left\{\frac{K_{4}+M K_{5}}{K_{0}}+\frac{M K_{4}\left(K_{2}+M^{\prime} K_{3}\right)}{K_{0}^{2}}\right\}\|f-g\| \\
& +\frac{M K_{6}}{K_{0}}\left\|f^{\prime}-g^{\prime}\right\|+\left\{\frac{1}{K_{0}}+\frac{M\left(K_{2}+M^{\prime} K_{3}\right)}{K_{0}^{2}}\right\}\|F-G\|_{1} \\
\leq & \varrho\|f-g\|_{1}+\left\{\frac{1}{K_{0}}+\frac{M\left(K_{2}+M^{\prime} K_{3}\right)}{K_{0}^{2}}\right\}\|F-G\|_{1} .
\end{aligned}
$$

Thus

$$
\|f-g\|_{1} \leq \frac{1}{1-\varrho}\left\{\frac{1}{K_{0}}+\frac{M\left(K_{2}+M^{\prime} K_{3}\right)}{K_{0}^{2}}\right\}\|F-G\|_{1},
$$

which proves the stability.

Now we give an example to illustrate the main theorem.

EXAMPLE 1. Consider the functional equation

$$
\sum_{i=1}^{\infty} \lambda_{i}(x) H_{i}\left(f^{i}(x)\right)=\frac{1}{2}\left[1+x-\cos \frac{\pi x}{2}\right], \quad \forall x \in[0,1],
$$

where

$$
\lambda_{1}(x)=1-\frac{1}{k}\left(e^{x}-1-x\right), \quad \lambda_{i}(x)=\frac{1}{k} \frac{x^{i}}{i !} \quad \text { for } i=2,3, \ldots,
$$

with $k=27 e^{9}, F(x)=\frac{1}{2}[1+x-\cos (\pi x / 2)] \in \mathcal{F}_{1 / 2}^{1}\left((\pi+2) / 4, \pi^{2} / 8\right), H_{1}(x)=$ $x \in \mathcal{F}_{1}^{1}(1,0)$ and $H_{i}(x)=x^{i-1} \in \mathcal{F}_{0}^{1}(i-1,(i-1)(i-2))$ for each $i \geq 2$.

Here $l_{1}=1, L_{1}=1, L_{1}^{\prime}=0, \gamma_{1}=1-(e-1) / k, \Lambda_{1}=1, \alpha_{1}=(e-1) / k$, $\beta_{1}=e / k$ and $l_{i}=0, L_{i}=i-1, L_{i}^{\prime}=(i-1)(i-2), \gamma_{i}=0$,

$$
\Lambda_{i}=\frac{1}{k} \frac{1}{i !}, \quad \alpha_{i}=\frac{1}{k} \frac{1}{(i-1) !}, \quad \beta_{i}=\frac{1}{k} \frac{1}{(i-2) !}
$$

for each $i \geq 2$. 
For $M=3$ and $\delta=1 / 4$ it is easily seen that the series $\sum_{i=1}^{\infty} \beta_{i}$, $\sum_{i=1}^{\infty} \Lambda_{i}\left\{L_{i}+L_{i}^{\prime}\right\} M^{2 i}$ and $\sum_{i=1}^{\infty} \alpha_{i} L_{i} M^{2 i}$ are convergent. Now

$$
\begin{aligned}
K_{1} & =\sum_{i=1}^{\infty}\left\{\frac{1}{\delta} \alpha_{i}+\Lambda_{i} L_{i} M^{i-1}\right\} \\
& =4\left\{\frac{e-1}{k}+\sum_{i=2}^{\infty} \frac{1}{k} \frac{1}{(i-1) !}\right\}+1+\frac{1}{k} \sum_{i=2}^{\infty} \frac{i-1}{i !} M^{i-1} \\
& \leq 4\left\{\frac{e-1}{k}+\frac{e-1}{k}\right\}+\frac{1}{k} \sum_{i=2}^{\infty} \frac{1}{(i-1) !} M^{i-1}+1 \\
& \leq \frac{8(e-1)}{k}+\frac{1}{k}\left(e^{M}-1\right)+1=\frac{1}{27 e^{9}}\left[8(e-1)+e^{3}-1\right]+1<2
\end{aligned}
$$

and hence $K_{1} \delta<1 / 2$. Moreover,

$$
\begin{aligned}
K_{0} & =\sum_{i=1}^{\infty} \gamma_{i} l_{i} \delta^{i-1}-\frac{1}{\delta} \sum_{i=1}^{\infty} \alpha_{i}=\gamma_{1}-\frac{1}{\delta} \sum_{i=1}^{\infty} \alpha_{i}=1-\frac{e-1}{k}-\frac{2(e-1)}{\delta k} \\
& =1-\frac{1}{k \delta}[(e-1)(2+\delta)]=1-\frac{4}{27 e^{9}}[(e-1)(2+1 / 4)]>2 / 3
\end{aligned}
$$

and

$$
\begin{aligned}
K_{3} & =\frac{1}{\delta^{3}} \sum_{i=1}^{\infty} \alpha_{i}+\frac{1}{M-1} \sum_{i=1}^{\infty} \Lambda_{i} L_{i} M^{i-2}\left(M^{i-1}-1\right) \\
& \leq 4^{3} \cdot \frac{2(e-1)}{k}+\frac{1}{k(M-1)} \sum_{i=2}^{\infty} \frac{i-1}{i !} M^{2 i-3} \\
& \leq \frac{2^{7}(e-1)}{k}+\frac{M}{k(M-1)} \sum_{i=2}^{\infty} \frac{1}{(i-2) !} M^{2(i-2)} \leq \frac{2^{7}(e-1)}{k}+\frac{M e^{M^{2}}}{k(M-1)} \\
& =\frac{2^{7}(e-1)}{27 e^{9}}+\frac{1}{27 e^{9}} \frac{3}{2} e^{9} \leq \frac{1}{2 \cdot 3^{3}}+\frac{1}{18}=\frac{2}{27}
\end{aligned}
$$

so that $M^{2} K_{3} \leq 2 / 3$. This implies that $K_{0}>M^{2} K_{3}$. As $K_{0} M>2$ and $K_{1} \delta<1 / 2$ we have $F \in \mathcal{F}_{1 / 2}^{1}\left((\pi+2) / 4, \pi^{2} / 8\right) \subseteq \mathcal{F}_{K_{1} \delta}^{1}\left(K_{0} M, M^{*}\right)$ where $M^{*}=\pi^{2} / 8$. It is easy to prove that $K_{2}<1$ and $K_{0}-M^{2} K_{3} \geq 1 / 4$. Thus by Theorem 3.2, the functional equation (3.32) has a solution $f$ in $\mathcal{F}_{0.25}^{1}\left(3, M^{\prime}\right)$ for every $M^{\prime} \geq 4\left(\pi^{2} / 8+9\right)$, since

$$
4\left(\frac{\pi^{2}}{8}+9\right)>\frac{M^{*}+M^{2} K_{2}}{K_{0}-M^{2} K_{3}} .
$$


We also deduce a corollary which provides a set of sufficient conditions for the existence of smooth solutions to the functional equation

$$
\sum_{i=1}^{n} \lambda_{i}(x) H_{i}\left(f^{i}(x)\right)=F(x) .
$$

Corollary 3.7. Let $\lambda_{i} \in \mathcal{Q}^{1}\left(\alpha_{i}, \beta_{i}\right)$ be nonnegative functions on I for each $i=1, \ldots, n$ such that $\gamma_{i} \leq \lambda_{i}(x) \leq \Lambda_{i}$ where $\alpha_{i}, \beta_{i}, \gamma_{i}$ and $\Lambda_{i}$ are nonnegative numbers for $i=1, \ldots, n$ and $\sum_{i=1}^{n} \lambda_{i}(x)=1$ for $x \in I$. Let $H_{i} \in \mathcal{F}_{l_{i}}^{1}\left(L_{i}, L_{i}^{\prime}\right)$ where $l_{i}, L_{i}, L_{i}^{\prime}$ are nonnegative numbers for $i=1, \ldots, n$. Let $0<\delta<1, M>1$ and $M^{*} \geq 0$. Define

$$
\begin{aligned}
& K_{0}=\sum_{i=1}^{n} \gamma_{i} l_{i} \delta^{i-1}-\frac{1}{\delta} \sum_{i=1}^{n} \alpha_{i}, \\
& K_{1}=\sum_{i=1}^{n}\left\{\frac{1}{\delta} \alpha_{i}+\Lambda_{i} L_{i} M^{i-1}\right\}, \\
& K_{2}=\sum_{i=1}^{n}\left\{\frac{1}{\delta^{2}} \beta_{i}+\frac{2}{\delta} \alpha_{i} L_{i} M^{i-1}+\Lambda_{i} L_{i}^{\prime} M^{2(i-1)}\right\}, \\
& K_{3}=\frac{1}{\delta^{3}} \sum_{i=1}^{n} \alpha_{i}+\sum_{i=1}^{n} \Lambda_{i} L_{i} M^{i-2} \frac{M^{i-1}-1}{M-1} .
\end{aligned}
$$

Suppose that $K_{0}>M^{2} K_{3}$. Then for any $F$ in $\mathcal{F}_{K_{1} \delta}^{1}\left(K_{0} M, M^{*}\right)$, the functional equation (3.33) has a solution $f$ in $\mathcal{F}_{\delta}^{1}\left(M, M^{\prime}\right)$ for every $M^{\prime} \geq$ $\left(M^{*}+M^{2} K_{2}\right) /\left(K_{0}-M^{2} K_{3}\right)$.

Proof. This follows directly from Theorem 3.2, upon choosing $\lambda_{i}(x)=0$ and $H_{i}(x)=0$ for $i>n$.

EXAMPle 2. Consider the functional equation

$$
4\left[(4242-x) f(x)+\left(f^{2}(x)\right)^{2}+x\left(f^{3}(x)\right)^{5}\right]=4243\left(3 x^{2}+x\right), x \in[0,1] .
$$

In order to apply Corollary 3.7, set $\lambda_{1}=(4242-x) / 4243, \lambda_{2}=1 / 4243$, $\lambda_{3}=x / 4243, H_{1}(x)=x, H_{2}(x)=x^{2}, H_{3}(x)=x^{5}$ and $F(x)=\frac{1}{4}\left(3 x^{2}+x\right)$. It can be easily seen that

$$
\begin{aligned}
& \gamma_{1}=\frac{4241}{4243}, \quad \Lambda_{1}=\frac{4242}{4243}, \quad \alpha_{1}=\frac{1}{4243}, \quad \beta_{1}=0, \quad l_{1}=1=L_{1}, \quad L_{1}^{\prime}=0 ; \\
& \gamma_{2}=\frac{1}{4243}=\Lambda_{2}, \quad \alpha_{2}=\beta_{2}=l_{2}=0, \quad L_{2}=2=L_{2}^{\prime} ; \\
& \gamma_{3}=0, \quad \Lambda_{3}=\frac{1}{4243}=\alpha_{3}, \quad \beta_{3}=0=l_{3}, \quad L_{3}=5, \quad L_{3}^{\prime}=20 .
\end{aligned}
$$

For $\delta=1 / 8, M=2$ and $M^{*}=3 / 2$, it is readily seen that

$$
K_{0}=\frac{4225}{4243}, \quad K_{1}=\frac{4282}{4243}, \quad K_{2}=\frac{664}{4243}, \quad K_{3}=\frac{1056}{4243} .
$$


Hence

$$
K_{0}-M^{2} K_{3}=\frac{4225}{4243}-4 \cdot \frac{1056}{4243}=\frac{1}{4243} \quad \text { and } \quad \frac{M^{*}+M^{2} K_{2}}{K_{0}-M^{2} K_{3}}=18041 / 2 .
$$

Clearly

$$
K_{1} \delta<\frac{1}{4} \leq F^{\prime}(x)=\frac{1}{4}(6 x+1) \leq \frac{7}{4} \leq K_{0} M .
$$

Thus $F \in \mathcal{F}_{K_{1} \delta}^{1}\left(K_{0} M, M^{*}\right)$.

By Corollary 3.7, the functional equation (3.34) has a solution $f$ in $\mathcal{F}_{1 / 8}^{1}\left(2, M^{\prime}\right)$ for every $M^{\prime} \geq 18041 / 2$.

The concluding corollary answers a question on the existence of smooth solution raised by Baker and Zhang [1].

Corollary 3.8. Let $\lambda_{i} \in \mathcal{Q}^{1}\left(\alpha_{i}, \beta_{i}\right)$ be nonnegative functions on I for each $i=1, \ldots, n$ such that $\gamma_{i} \leq \lambda_{i}(x) \leq \Lambda_{i}$ where $\alpha_{i}, \beta_{i}, \gamma_{i}$ and $\Lambda_{i}$ are nonnegative numbers for $i=1, \ldots, n$ and $\sum_{i=1}^{n} \lambda_{i}(x)=1$ for $x \in I$. Let $0<\delta<1, M>1$ and $M^{*} \geq 0$. Define

$$
\begin{aligned}
& K_{0}=\sum_{i=1}^{n} \gamma_{i} \delta^{i-1}-\frac{1}{\delta} \sum_{i=1}^{n} \alpha_{i}, \\
& K_{1}=\sum_{i=1}^{n}\left\{\frac{1}{\delta} \alpha_{i}+\Lambda_{i} M^{i-1}\right\} \\
& K_{2}=\sum_{i=1}^{n}\left\{\frac{1}{\delta^{2}} \beta_{i}+\frac{2}{\delta} \alpha_{i} M^{i-1}\right\} \\
& K_{3}=\frac{1}{\delta^{3}} \sum_{i=1}^{n} \alpha_{i}+\sum_{i=1}^{n} \Lambda_{i} M^{i-2} \frac{M^{i-1}-1}{M-1} .
\end{aligned}
$$

Suppose further that $K_{0}>M^{2} K_{3}$. Then for any $F$ in $\mathcal{F}_{K_{1} \delta}^{1}\left(K_{0} M, M^{*}\right)$, the functional equation

$$
\sum_{i=1}^{n} \lambda_{i}(x) f^{i}(x)=F(x)
$$

has a solution $f$ in $\mathcal{F}_{\delta}^{1}\left(M, M^{\prime}\right)$ for every $M^{\prime} \geq\left(M^{*}+M^{2} K_{2}\right) /\left(K_{0}-M^{2} K_{3}\right)$.

Proof. This follows directly from Corollary 3.7, upon choosing $H_{i}(x)=x$ for $i=1, \ldots, n$.

Acknowledgements. The authors are highly thankful to the referee for his critical remarks and suggestions resulting in a much improved version of the work. Further, V. Murugan acknowledges the financial support in the form of a Senior Research Fellowship from the Council of Scientific and Industrial Research (CSIR), India for carrying out this research. 


\section{References}

[1] J. A. Baker and W. Zhang, Continuous solutions for a polynomial-like iterative equation with variable coefficients, Ann. Polon. Math. 73 (2000), 29-36.

[2] M. Kuczma, B. Choczewski and R. Ger, Iterative Functional Equations, Encyclopedia Math. Appl. 32, Cambridge Univ. Press, Cambridge, 1990.

[3] M. Kulczycki and J. Tabor, Iterative functional equations in the class of Lipschitz functions, Aequationes Math. 64 (2002), 24-33.

[4] V. Murugan and P. V. Subrahmayam, Existence of solutions for equations involving iterated functional series, Fixed Point Theory Appl. 2005, no. 2, 219-232.

[5] J. Si, $C^{2}$ solutions of the iterated equation $\sum_{i=1}^{n} \lambda_{i} f^{i}(x)=F(x)$, Acta Math. Sinica 36 (1993), 348-357 (in Chinese).

[6] J. Si and W. Zhang, On $C^{2}$ solutions of a functional equation, Acta Math. Sinica (N.S.) 14 (1998), suppl., 705-710.

[7] J. Tabor and M. Żołdak, Iterative equations in Banach spaces, J. Math. Anal. Appl. 299 (2004), 651-662.

[8] W. Zhang, Stability of the solution of the iterated equation $\sum_{i=1}^{n} \lambda_{i} f^{i}(x)=F(x)$, Acta Math. Sci. 8 (1988), 421-414.

[9] - Discussion on the differentiable solutions of the iterated equation $\sum_{i=1}^{n} \lambda_{i} f^{i}(x)=$ $F(x)$, Nonlinear Anal. 15 (1990), 387-398.

Department of Mathematics

Indian Institute of Technology Madras

Chennai 600 036, India

E-mail: pvs@iitm.ac.in

Received 6.5.2007

and in final form 20.9.2007 